\title{
Clinical findings in families with chordoma with and without $T$ gene duplications and in patients with sporadic chordoma reported to the Surveillance, Epidemiology, and End Results program
}

\author{
Dilys M. Parry, PhD, ${ }^{1}$ Mary L. McMaster, MD, ${ }^{1,2}$ Norbert J. Liebsch, MD, PhD, ${ }^{3}$ \\ Nicholas J. Patronas, MD, ${ }^{4}$ Martha M. Quezado, MD, ${ }^{5}$ Deborah Zametkin, MSN, ${ }^{6}$ \\ Xiaohong R. Yang, PhD, ${ }^{1}$ and Alisa M. Goldstein, PhD ${ }^{1}$ \\ 'Division of Cancer Epidemiology \& Genetics, National Cancer Institute, NIH, Department of Health and Human Services, \\ Bethesda; ${ }^{2}$ Commissioned Corps of the United States Public Health Service, Bethesda, Maryland; ${ }^{3}$ Department of Radiation \\ Oncology, Massachusetts General Hospital, Boston, Massachusetts; ${ }^{4}$ Radiology and Imaging Sciences, NIH Clinical Center,

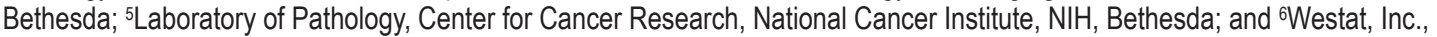 \\ Rockville, Maryland
}

OBJECTIVE To gain insight into the role of germline genetics in the development of chordoma, the authors evaluated data from 2 sets of patients with familial chordoma, those with and without a germline duplication of the $T$ gene ( $T$-dup+ vs $T$-dup-), which was previously identified as a susceptibility mechanism in some families. The authors then compared the patients with familial tumors to patients with sporadic chordoma in the US general population reported to the National Cancer Institute's Surveillance, Epidemiology, and End Results (SEER) program through 2015.

METHODS Evaluation of family members included review of personal and family medical history, physical and neurological examination, and pre- and postcontrast MRI of the skull base and spine. Sixteen patients from 6 white families with chordoma had a chordoma diagnosis at family referral. Screening MR images of 35 relatives revealed clival lesions in 6, 4 of which were excised and confirmed to be chordoma. Thus, data were available for 20 patients with histologically confirmed familial chordoma. There were 1759 patients with histologically confirmed chordoma in SEER whose race was known.

RESULTS The median age at chordoma diagnosis differed across the groups: it was lowest in T-dup+ familial patients (26.8 years, range 5.3-68.4 years); intermediate in T-dup- patients (46.2 years, range 11.8-60.1 years); and highest in SEER patients (57 years, range 0-98 years). There was a marked preponderance of skull base tumors in patients with familial chordoma (93\% in T-dup+ and $83 \%$ in T-dup-) versus 38\% in the SEER program (37\% in white, $53 \%$ in black, and $48.5 \%$ in Asian/Pacific Islander/American Indian/Alaska Native patients). Furthermore, $29 \%$ of white and $16 \%-17 \%$ of nonwhite SEER patients had mobile-spine chordoma, versus no patients in the familial group. Several T-dup+familial chordoma patients had putative second/multiple primary chordomas.

CONCLUSIONS The occurrence of young age at diagnosis, skull base presentation, or multiple primary chordomas should encourage careful review of family history for patients diagnosed with chordoma as well as screening of at-risk family members by MRI for early detection of chordoma. Furthermore, given genetic predisposition in some patients with familial chordoma, identification of a specific mutation in a family will permit surveillance to be limited to mutation carriers-and consideration should be given for imaging the entire neuraxis in any chordoma patient presenting at an early age or with a blood relative with chordoma. Finally, future studies should explore racial differences in age at diagnosis and presenting site in chordoma.

https://thejns.org/doi/abs/10.3171/2020.4.JNS193505

KEYWORDS chordoma; familial; $T$ (brachyury); skull base; oncology

ABBREVIATIONS A/PI/AI/AN = Asian/Pacific Islander/American Indian/Alaska Native; BNCT = benign notochordal cell tumor; GTR = gross-total resection; NIH = National Institutes of Health; RT = radiation therapy; SEER = Surveillance, Epidemiology, and End Results.

SUBMITTED December 27, 2019. ACCEPTED April 6, 2020.

INCLUDE WHEN CITING Published online June 19, 2020; DOI: 10.3171/2020.4.JNS193505. 
$\mathrm{C}$ HORDOMA is a rare bone cancer $(<1$ per 100,000$)$ that is believed to arise from remnants of the notochord that persist along the axial skeleton into adulthood. Chordoma occurs more frequently in males than females and in Caucasians than African Americans. ${ }^{1,2}$ Chordoma incidence increases with age and is rare in young patients, especially in the first decade of life. The usual sites of origin are the skull base, mobile spine, and sacrum/coccyx. Although most chordomas are sporadic, 10 multiple-case families with at least 2 blood relatives with histopathologically confirmed chordoma have been reported. ${ }^{3-8} \mathrm{We}$ previously identified autosomal dominant inheritance of a duplication of the $T$ gene (brachyury) by using a wholegenome human array-comparative genomic hybridization (array-CGH) chip followed by a quantitative polymerase chain reaction assay for confirmation in 4 of 7 families investigated. ${ }^{8}$

To gain insight into the role of germline genetics in the development of chordoma and to evaluate whether any clinical features might impact surveillance or screening, we evaluated clinical data from 2 sets of patients with familial chordoma: those with a germline duplication of the $T$ gene $(T-d u p+)$ and those without a $T$ gene duplication (T-dup-). We then compared the patients with familial chordoma to those with sporadic chordoma in the US general population reported to the National Cancer Institute's
Surveillance, Epidemiology, and End Results (SEER) program through 2015.

\section{Methods}

\section{Chordoma Families}

We ascertained 7 families, all Caucasian, with $\geq 2$ blood relatives with histopathologically confirmed chordoma through physician referral (families 1, 3, 4, and 7), self-referral (families 6 and 8), and evaluation of patients reported to 4 SEER registries between 1988 and 1998 (family 2). We excluded family 3 because of limited clinical information on the chordoma patients. Therefore, this report focuses on 6 families (Fig. 1).

Evaluated individuals included living family members with chordoma and selected first- and second-degree relatives. This study was approved by the institutional review board of the National Cancer Institute, National Institutes of Health (NIH). Patients gave written informed consent or assented before undergoing evaluation. Families were seen at the NIH Clinical Center. Some affected members of families 2, 4, 6, and 7 were also seen at Massachusetts General Hospital (by N.J.L.). Molecular genetic studies had previously revealed duplication of the $T$ gene in families 1,4 , and $8.6,8$

At the NIH Clinical Center, evaluation, regardless of
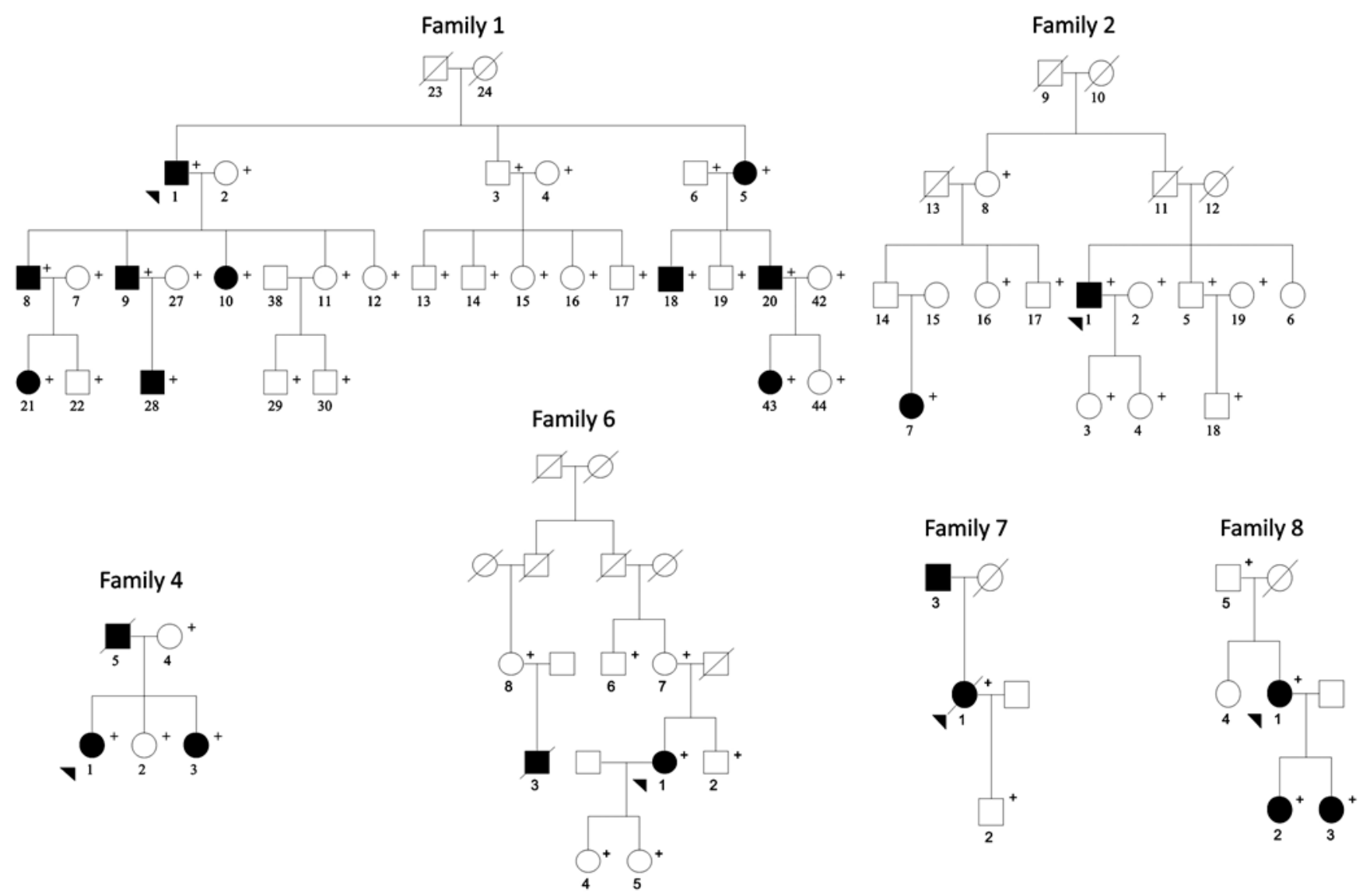

FIG. 1. Chordoma families. Pedigrees for the 6 families (families 1, 2, 4, 6, 7, and 8) in the current study. Filled squares and circles indicate a patient diagnosed with chordoma. Plus signs show the family members who were participants in the clinical study. 
chordoma status, included review of personal and family medical history, physical and neurological examination, and pre- and postcontrast MRI of the skull base and spine including sacrum and coccyx. All chordoma diagnoses were confirmed by review of pathology materials and/ or reports and medical records. Follow-up data were obtained during return clinic visits, review of outside medical records and pathology reports, and/or telephone conversations with patients or relatives.

\section{SEER Program}

We used SEER to obtain population-based data for all histologically confirmed cases of chordoma among patients reported to 18 cancer registries during the period 1973$2015^{9}$ for comparison with familial chordoma patients. We identified cases using the WHO's International Classification of Diseases for Oncology, Third Edition (ICD-O-3) morphology code for chordoma (9370/3) and the ICD-O-2 and ICD-O-3 topography codes for sites of presentation. ${ }^{1}$ Sixteen cases in which race was unknown were excluded from the analyses. Because the scope of SEER data collection and reporting has varied over time, we limited analysis of each variable to the number of patients with known status. Data were analyzed using SEER*Stat v.8.3.5. ${ }^{10}$

\section{Statistical Analysis}

For categorical variable comparisons (e.g., presenting site of chordoma), the Pearson chi-square or Fisher exact test was used depending on sample sizes. Because the incidence rates, distribution of chordomas at presenting sites, and ages at diagnosis of chordoma differed by race, and almost $90 \%$ of patients with this rare tumor and all members of the studied chordoma families were white, analyses of familial versus SEER chordoma patients were restricted to white chordoma patients. Because of the relatively small number of patients available for analysis, we assumed independence of patients with chordoma within families.

\section{Results \\ Chordoma Families}

Fifty-one individuals from 6 chordoma families were study participants (Table 1, Fig. 1). Sixteen patients had a diagnosis of chordoma at referral. Of these, 11 chordoma patients underwent MRI evaluation: skull base and spine ( $\mathrm{n}=8$ skull base chordomas and 1 sacral chordoma), and spine ( $\mathrm{n}=2$ skull base chordomas). Thirty-five blood relatives who enrolled in the study without a diagnosis of chordoma were screened by MRI: skull base and spine (n =34) and skull base only $(n=1)$.

\section{Screening MR Images of Relatives}

Screening MR images of relatives revealed clival lesions in 6 , all from family 1 . Four lesions were excised and confirmed to be chordoma (Table 1). The 2 other clival lesions were not biopsied; therefore, although their location and imaging characteristics were compatible with chordoma, no diagnosis could be confirmed. Thus, data were available for 20 histologically confirmed chordoma patients. For ease of presentation, T-dup+ (3 families, 14 patients) and $T$-dup- (3 families, 6 patients) families are presented separately (Tables $1-3$, Supplemental Table 1 ).

\section{Clinical Findings, Treatment, and Outcome in T-dup+ Families}

Among the 14 chordoma patients from $T$-dup + families, 13 presented with skull base tumors; 1 presented with sacrococcygeal chordoma (Table 2). Among the 13 skull base chordomas, 8 occurred in females (61\%) and 5 (39\%) in males, with a median age at diagnosis of 25.5 years (range 5.3-68.4 years). All 13 skull base chordomas arose from the clivus. In most, there was a soft-tissue component that included only the nasopharynx. Two tumors were more extensive (Table 3, Supplemental Table 1).

Of the 11 measured skull base chordomas, 6 were diagnosed because of presenting symptoms (median diameter

TABLE 1. Patients and relatives included in the familial chordoma study

\begin{tabular}{|c|c|c|c|c|c|c|c|}
\hline \multirow{3}{*}{$\begin{array}{l}\text { Family } \\
\text { ID }\end{array}$} & \multirow{3}{*}{$\begin{array}{l}\text { T-dup+ } \\
\text { Present }\end{array}$} & \multicolumn{3}{|c|}{$\begin{array}{c}16 \text { Patients Diagnosed w/ } \\
\text { Chordoma Before Study Entry }\end{array}$} & \multicolumn{3}{|c|}{$\begin{array}{c}\text { Clinical Findings in } 35 \text { Relatives Screened at } \\
\text { NIH by MRI }\end{array}$} \\
\hline & & \multirow[b]{2}{*}{ Imaged at $\mathrm{NIH}$} & \multicolumn{2}{|c|}{ Not Imaged at NIH } & \multirow[b]{2}{*}{ Chordoma } & \multirow{2}{*}{$\begin{array}{l}\text { Another Abnormal } \\
\text { Finding }\end{array}$} & \multirow{2}{*}{$\begin{array}{l}\text { No Abnorma } \\
\text { Findings }\end{array}$} \\
\hline & & & Alive & Deceased & & & \\
\hline 1 & Yes & 4 & 0 & 0 & 4 & $2^{*}$ & 13 \\
\hline 2 & No & 1 & 1 & 0 & 0 & $1 \dagger$ & 6 \\
\hline 4 & Yes & 2 & 0 & 1 & 0 & 0 & $1 \ddagger$ \\
\hline 6 & No & 1 & 0 & 1 & 0 & 0 & 5 \\
\hline 7 & No & 0 & 2 & 0 & 0 & 0 & 1 \\
\hline 8 & Yes & 3 & 0 & 0 & 0 & 0 & 2 \\
\hline Totals & & 11 & 3 & 2 & 4 & 3 & 28 \\
\hline
\end{tabular}

* Both relatives had MRI findings suggestive of clival chordoma, but neither lesion was biopsied.

† This relative had a cystic lesion within her cervical spinal cord at age 16 years, which was detected on the screening MR images. It was resected and the histopathological diagnosis was juvenile pilocytic astrocytoma.

$\ddagger$ This relative had been diagnosed elsewhere at age 11 years with a cerebellar astrocytoma, which was resected. When she was seen at $\mathrm{NIH}$, no abnormal MRI findings were detected. 


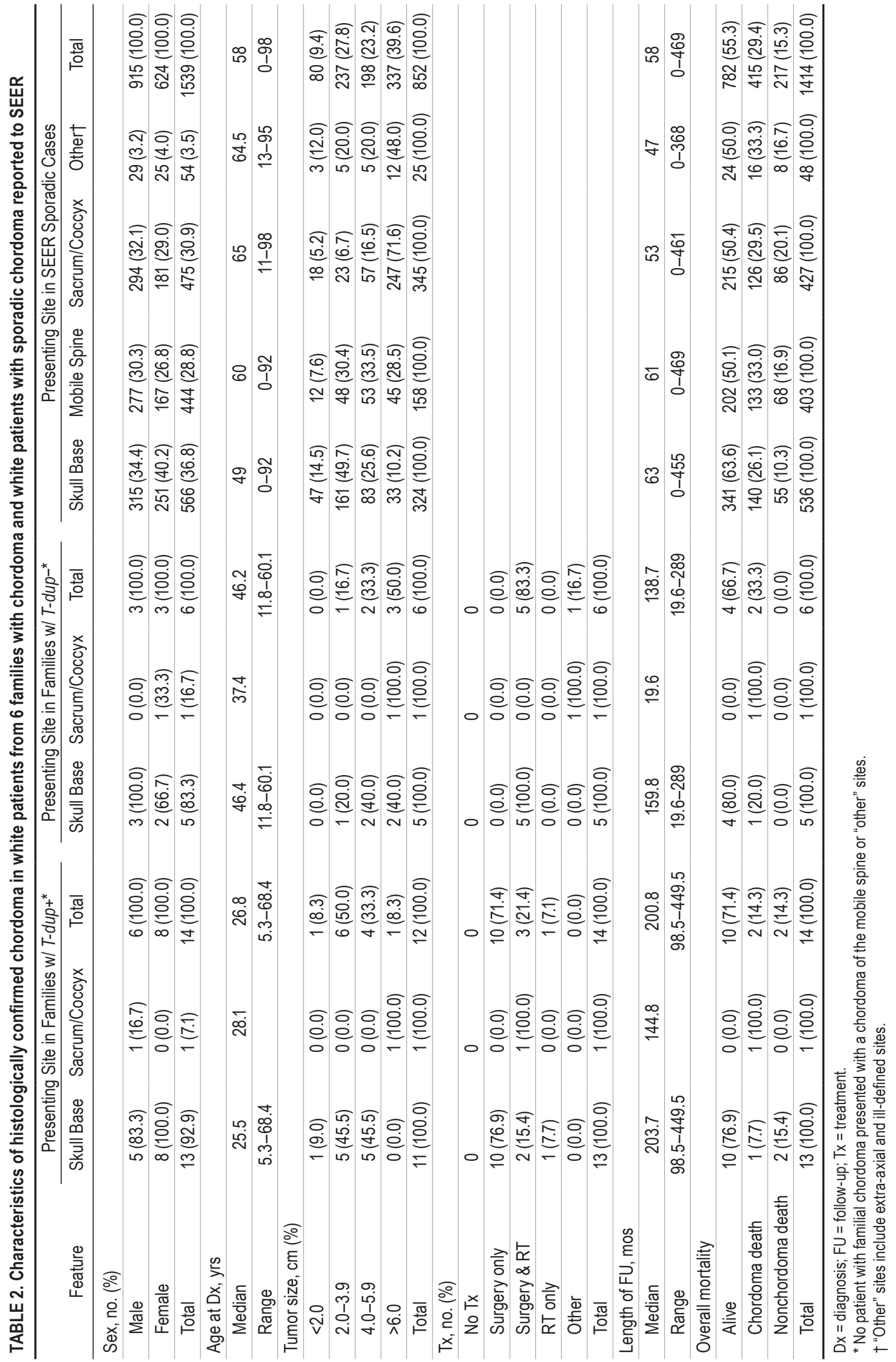


TABLE 3. Location, extension, size, and initial treatment of chordoma in 20 patients from 6 chordoma families

\begin{tabular}{|c|c|c|c|c|c|}
\hline Family & $\begin{array}{l}\text { No. of } \\
\text { Pts }\end{array}$ & Tumor Location \& Extension (cranial tumors) & $\begin{array}{l}\text { Size } \\
(\mathrm{cm})\end{array}$ & Major Symptoms & Initial $T x^{*}$ \\
\hline \multicolumn{6}{|l|}{ T-dup+ } \\
\hline \multirow[t]{4}{*}{ Family 1} & $2 \dagger$ & Clivus, nasopharynx & $2.5,3.0$ & Snoring, nasal obstruction & GTR \\
\hline & $2 \ddagger$ & Clivus, nasopharynx & $3.0,4.9$ & Nasal speech \& obstruction, snoring $†$ & $\mathrm{RR}$ \\
\hline & $2 \S$ & Clivus, nasopharynx & $2.0,2.4$ & None & $\mathrm{RR}$ \\
\hline & $1 \dagger$ & $\begin{array}{l}\text { Clivus, sphenoid sinus, cavernous sinus, Meckel's } \\
\text { cave, petrous apex }\end{array}$ & 5.4 & Headaches, nausea, vomiting & $P R+R T$ \\
\hline \multirow[t]{3}{*}{ Family 4} & $1 \dagger$ & Clivus & NA & Nasal congestion \& obstruction & $B x+R T$ \\
\hline & $1 \dagger$ & Clivus & 4 & Nasal congestion \& obstruction & STR + RT \\
\hline & $1 \dagger$ & Clivus, sphenoid sinus, nasopharynx & 4.5 & Ear pain & PR \\
\hline \multirow[t]{3}{*}{ Family 8} & $1 \dagger$ & Clivus, nasopharynx & 3.9 & Epistaxis, nasal congestion, obstruction & $\mathrm{RR}$ \\
\hline & $1 \rrbracket$ & Clivus & NA & Nasal congestion, obstructed breathing & RR \\
\hline & $1^{* *}$ & Clivus & 1.5 & None & $\mathrm{RR}$ \\
\hline Total & 13 & & & & \\
\hline \multicolumn{6}{|l|}{ T-dup- } \\
\hline \multirow[t]{2}{*}{ Family 2} & $1 \dagger$ & $\begin{array}{l}\text { Clivus, nasal cavity; paranasal, ethmoid sphenoid, \& } \\
\text { maxillary sinuses; nasopharynx }\end{array}$ & $>6.0$ & Nasal congestion \& obstruction & $P R+R T$ \\
\hline & $1 \dagger$ & Clivus, pons & 3 & Diplopia, headaches, rt pupil > It, rt ptosis & GTR + RT \\
\hline \multirow[t]{2}{*}{ Family 6} & $1 \dagger$ & Clivus, foramen magnum to $\mathrm{C} 2$ vertebra & 4.2 & Headaches from occipital to frontal region & $P R+R T$ \\
\hline & $1 \dagger$ & Clivus w/ sinus-nasal extension & $>6.0$ & $\begin{array}{l}\text { Aural fullness, bilat ptosis, vertical diplopia, } \\
\text { nasal obstruction, epistaxis }\end{array}$ & $P R+R T$ \\
\hline Family 7 & $1 \dagger$ & Clivus, craniocervical junction & $\geq 5.0$ & $\begin{array}{l}\text { Atrophy rt tongue; pain rt neck, shoulder, \& } \\
\text { arm; numbness \& tingling, rt hand }\end{array}$ & $S T R+R T$ \\
\hline Total & 5 & & & & \\
\hline Family & $\begin{array}{l}\text { No. of } \\
\text { Pts }\end{array}$ & Tumor Location \& Extension (sacrococcygeal tumors) & $\begin{array}{l}\text { Size } \\
(\mathrm{cm})\end{array}$ & Major Symptoms & Initial Tx* \\
\hline \multicolumn{6}{|l|}{ T-dup+ } \\
\hline Family 1 & $1 \dagger$ & Coccyx to S3 vertebra, presacral region & $\geq 9.5$ & Buttock pain on sitting & GTR + RT \\
\hline \multicolumn{6}{|l|}{ T-dup- } \\
\hline Family 7 & $1 \dagger$ & $\begin{array}{l}\text { S3-5 vertebrae, presacral region, paraspinal soft } \\
\text { tissue, piriformis muscle }\end{array}$ & $\geq 10.0$ & Pain in It buttock, sacrum, lower back & $\mathrm{Bx}+$ palliative $\mathrm{RT}$ \\
\hline
\end{tabular}

$\mathrm{Bx}=$ biopsy; $\mathrm{NA}=$ not available; $\mathrm{PR}=$ partial resection; $\mathrm{Pts}=$ patients; $\mathrm{RR}$ = radical resection; $\mathrm{STR}=$ subtotal resection.

* Initial treatment denotes treatment within 4 months of diagnosis.

$\dagger$ These patients were diagnosed because they presented with symptoms.

$\ddagger$ These 2 patients reported long-standing symptoms but did not pursue a cause for them because their development had been so gradual. Subsequently, they were found to have skull base chordomas based on the NIH screening MR images.

$\S$ These patients were asymptomatic and diagnosed based on the NIH screening MR images.

I This patient was presumed to have enlarged tonsils and adenoids prior to initial surgery, so no preoperative images are available.

** This patient was asymptomatic but her local doctor had her screened by neuraxis MR images because her mother and younger sister had been diagnosed with skull base chordomas.

$4.2 \mathrm{~cm}$, range $2.5-5.4 \mathrm{~cm}$ ) and these tumors were larger than the 5 chordomas identified through MRI screening (median diameter $2.4 \mathrm{~cm}$, range $1.5-4.9 \mathrm{~cm}$ ) (see Table 3 and Supplemental Table 1 for symptom details). Only 1 chordoma measured $<2.0 \mathrm{~cm}$; it was diagnosed in an asymptomatic child (case 8-3) who was screened because of her chordoma family history. For patients diagnosed because of symptoms, the median time between symptom onset and diagnosis was 24 months (range 3 weeks-48 months).

The patient with $T$-dup+ sacrococcygeal chordoma was a man diagnosed at 28 years. He presented with buttock pain and had an 18-month delay between symptom onset and diagnosis (Table 3). His tumor originated in the midcoccyx, was approximately $9.5 \mathrm{~cm}$ in maximal diameter, and extended into the retrorectal and presacral space.

Neuroaxis MRI studies conducted in 13 patients revealed 2 with lesions suspected of being additional primary chordomas. Five $(38.5 \%)$ also had $\geq 1$ lesion with imaging findings consistent with benign notochordal cell tumor (BNCT). Details of the other relevant clinical findings are reported in Supplemental Table 1 and Supplemental Clinical Note.

All T-dup+ patients with primary skull base chordo- 
mas were treated initially with surgery; $10(76.9 \%)$ had no additional therapy and $3(23.1 \%)$ received adjuvant external-beam radiation therapy (RT) (Table 3 ). The patient with sacrococcygeal chordoma underwent gross-total resection (GTR) of extensive but localized tumor. Because microscopic tumor was present at surgical margins and in rectal tissue, the region was subsequently treated with adjuvant fractionated radiation.

After a median follow-up of 203.7 months, 10 (76.9\%) patients with skull base chordoma were alive at last contact (Table 2, Supplemental Table 1). Among these patients, 7 had no evidence of disease, 2 had stable tumor, and 1 developed pulmonary metastases without local recurrence 26.6 years after initial diagnosis. Three patients with $T$ $d u p+$ skull base chordoma have died, with 1 death directly attributable to chordoma. The patient (case 4-5) developed a recurrence in the paranasal sinuses 31 years after initial diagnosis and treatment at the age of 8 years. The tumor progressed despite serial resections, and the patient died of recurrent disease at the age of 47 years.

The patient with sacrococcygeal chordoma had widespread metastases when seen at the NIH. His disease progressed despite sequential treatment with multiple courses of different single chemotherapeutic agents, and he died at the age of 40 years, 144 months after diagnosis.

Table 4 shows clinical findings from 3 other screened $T$ - $d u p+$ relatives. Initial screening MRI identified asymptomatic clival lesions in 2 patients (cases 1-8 and 1-43) at ages 44 and 6 years, respectively. The third individual (case 1-44), the younger sister of case 1-43, had no abnormalities suggestive of chordoma on screening MRI at 3 years. At 13 years, a neck CT scan to evaluate a symptomatic thyroglossal duct cyst unexpectedly revealed a $1.6-\mathrm{cm}$ nasopharyngeal mass associated with bony erosion of the clivus. None of these clival lesions have been biopsied or excised.

\section{Clinical Findings, Treatment, and Outcome in T-dup- Families}

We evaluated 6 chordoma patients from $3 T$-dup - families (families 2, 6, and 7; Fig. 1, Table 1) in which no other chordoma-related mutations have been identified. Chordoma was diagnosed in 2 blood relatives in each family; males and females were equally affected.

The skull base was the presenting site for 5 patients; the sixth patient had a primary sacrococcygeal chordoma (Table 3, Supplemental Table 1). The median age at diagnosis of the patients with skull base chordoma was 46.4 years. All 5 skull base chordomas originated in the clivus but extended beyond it in anterior, superior, and/or posterior directions. All patients sought medical attention because of symptoms, which in 3 patients affected cranial nerves. The median time from initial symptoms to diagnosis was 7 months (range 1-36 months).

The 5 T-dup - skull base chordomas had a median diameter of $5.0 \mathrm{~cm}$ (range $3.5-12.8 \mathrm{~cm}$ ). All were locally advanced and treated with RT following surgery. After a median of 159.8 months, 4 patients were alive at last follow-up. Two had no evidence of disease, 1 had stable tumor, and 1 had local tumor progression to his optic nerve 24 years after initial diagnosis. The death of the patient

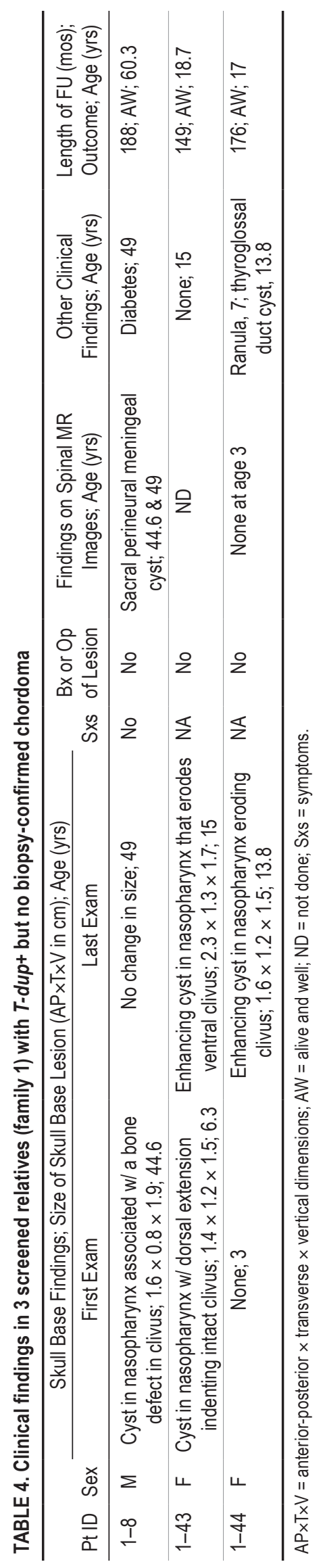


(case 6-3) was directly attributable to his tumor (Supplemental Table 1).

The patient with T-dup- sacrococcygeal chordoma was diagnosed at age 37 years, 98 months following her initial presentation with buttock pain (Table 3 ). Pulmonary metastases were found shortly after diagnosis. The tumor was treated with palliative high-dose proton-photon RT. She died at age 39 years from widely disseminated disease 19 months after diagnosis.

Spinal MRI studies were available in 2 of 6 T-duppatients. Neither had a lesion suspected of being a second primary chordoma or BNCT.

Ten families with at least 2 blood relatives with histopathologically confirmed chordoma have been described worldwide. ${ }^{3-8,11-18}$ The 6 families described in this report (listed by number in Table 5) include 3 of the 4 published $T$-dup + chordoma families. The fourth $T$-dup + chordoma family (family 3) has branches in both South Africa and England; we present the 2 branches separately but treat them as a single family. Family 3 displayed characteristics like those for the other $T$-dup + families: $\geq 3$ affected first-degree relatives, an autosomal dominant pattern, preponderance of skull base tumors, young ages at diagnosis (e.g., ages 3 and 8 years), and a member with putative multifocal chordoma diagnosed in the skull base and sacrum. ${ }^{3}$ Among the 3 additional reported chordoma families in Table 5, one is notable for the occurrence of sacral chordomas in 2 adult siblings, another features skull base chordomas in 2 very young brothers, and the third describes skull base chordomas in 2 adult siblings and 2 of their children. Geographically, the chordoma families were from the US, Italy, South Africa/England, Russia, and China. Overall, among the 34 confirmed cases of familial chordoma in the 10 families the male/female ratio was 15:19 (1:1.3), with 30 skull base tumors versus 4 sacral chordomas. The Chinese family showed a clinical pattern of chordoma similar to the $4 T$-dup + families, but mutation analysis results have not been reported.?

\section{Population-Based Chordomas Reported to SEER}

Supplemental Table 2 shows the clinical characteristics of 1759 histologically confirmed chordomas in patients with known race. Briefly, the overall incidence rate was 0.10 per 100,000 . Males comprised $59.1 \%$ of cases, resulting in a male/female ratio of 1.45:1. Most patients (87\%) were white. There were significant differences in the distribution of chordoma presenting site by race. Black and Asian/Pacific Islander/American Indian/Alaska Native (A/ $\mathrm{PI} / \mathrm{AI} / \mathrm{AN}$ ) patients had higher frequencies of skull base chordomas ( $\mathrm{p}=0.015$ and $\mathrm{p}=0.003$, respectively) and lower frequencies of mobile-spine chordomas $(\mathrm{p}=0.032$ and $\mathrm{p}$ $<0.0001$, respectively) compared to white patients. Overall, the median age at chordoma diagnosis was 57 years (range 0-98 years), but nonwhite patients had lower median ages at chordoma diagnosis versus white patients (Supplemental Table 2). Chordoma was uncommon at younger ages; only $287(16.3 \%)$ cases were diagnosed before the age of 35 years. However, a higher proportion of black (29.8\%) and $\mathrm{A} / \mathrm{PI} / \mathrm{AI} / \mathrm{AN}(23.9 \%)$ patients were diagnosed before 35 years compared to whites $(15.0 \%)(\mathrm{p}=0.002$, blacks vs whites; and $\mathrm{p}=0.003, \mathrm{~A} / \mathrm{PI} / \mathrm{AI} / \mathrm{AN}$ vs whites).

\section{Comparison of Familial Chordomas With Population- Based Chordomas Reported to SEER}

Table 2 shows the 1539 SEER-reported white patients with chordoma for comparison with the familial chordoma patients. Familial chordoma patients were much more likely than patients with sporadic chordoma in the US general population to present with skull base tumors: $18 / 20(90 \%)$ versus 566/1539 (36.8\%), respectively ( $\mathrm{p}<$ 0.0001). In contrast, $29 \%$ of SEER chordoma patients, but no familial chordoma patients, presented with chordoma in the mobile spine ( $\mathrm{p}<0.0001) . T-d u p+$ familial chordoma patients were diagnosed at much younger ages (median 26.8 years) than patients in either $T$-dup- families (median 46.2 years) or SEER chordoma patients (median 58 years). Moreover, $77 \%$ of the $T$-dup + skull base tumors were diagnosed by 35 years versus only $20 \%$ of $T$-dup- (p $=0.048$, vs $T$-dup + ) and $23.5 \%$ of SEER ( $p<0.0001$, vs $T$-dup+) skull base tumors. Several T-dup+ familial chordoma patients had putative second or multiple primary chordomas and/or BNCTs in the spine. No such clinical findings were observed in the small number of $T$-dup- familial chordoma patients available for examination. It was not possible to evaluate SEER chordoma patients for these features.

\section{Discussion}

To gain insight into the role of germline genetics in the development of chordoma, we evaluated data from 2 sets of familial chordoma patients, those with a germline duplication of the $T$ gene (T-dup+) and those without a $T$ gene duplication (T-dup-). We then compared the clinical data on familial chordoma patients with data from populationbased sporadic chordoma patients diagnosed in the US between 1973 and 2015. The results demonstrate that $T$-dup+ familial chordoma patients differ from sporadic chordoma patients (SEER program) in at least 2 aspects: the marked preponderance among the former of skull base tumors and the young age at which these tumors were diagnosed. Skull base tumors were also more frequent among T-dupfamilial chordoma patients; their age at diagnosis, however, did not differ markedly from that in sporadic chordoma patients. Among the chordoma patients reported to SEER, nonwhite SEER patients had a higher frequency of skull base chordomas, a lower frequency of mobile-spine chordomas, and younger age at diagnosis compared with white SEER chordoma patients.

In the general population, skull base chordoma was rare (3.7\%) in young patients ( $<10$ years). ${ }^{1}$ In contrast, 4 (31\%) $T$-dup + patients were diagnosed with skull base chordomas before 10 years of age, 3 of which were symptomatic, suggesting that these tumors have very early onset and/or unusually rapid growth. In fact, most skull base chordomas in T-dup+ patients occurred early, with almost $77 \%$ of the $T$-dup + skull base tumors diagnosed in patients by 35 years of age. In contrast, only about $26 \%$ of skull base tumors in SEER (23\% in whites, $40 \%$ in blacks, and 39\% in A/PI/AI/AN) and $20 \%$ of T-dup - skull base tumors were diagnosed by age 35 years.

The site of chordoma presentation varied with age. In both the familial and sporadic (SEER) settings, the earlier 


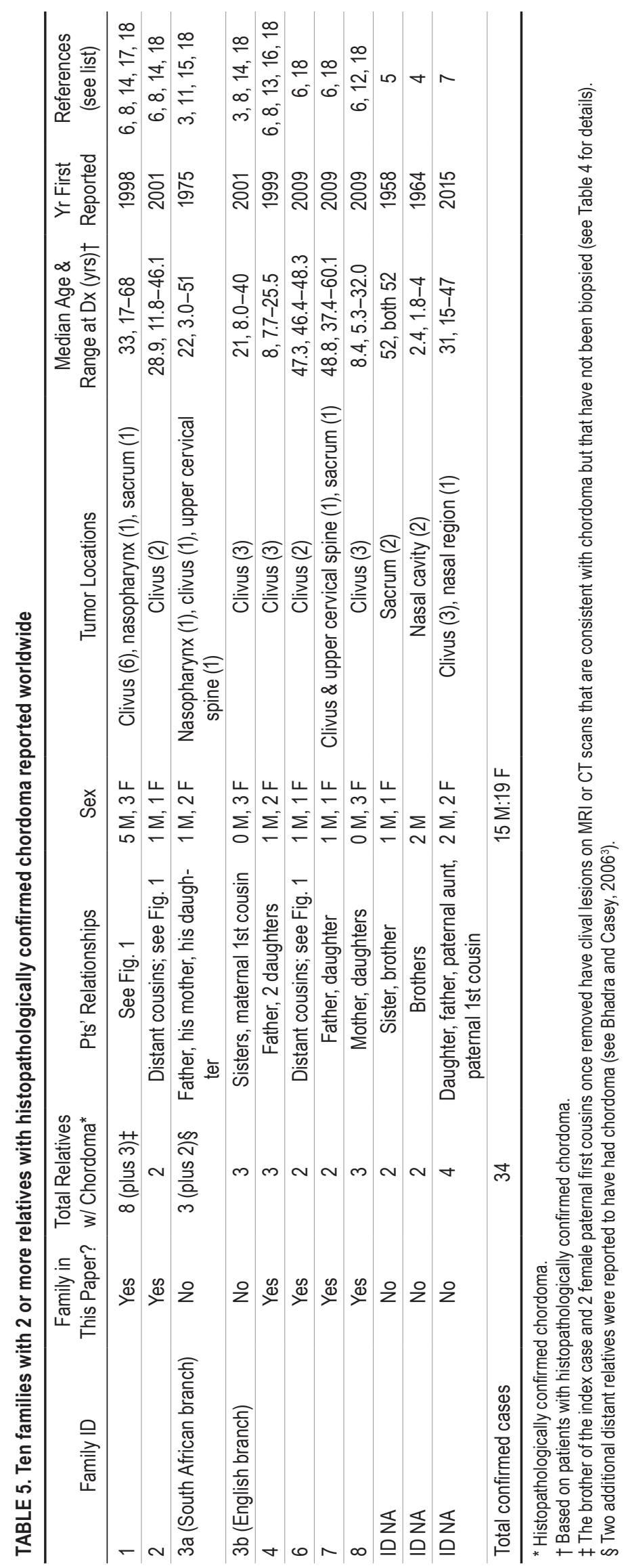


presentation of skull base versus sacrococcygeal chordomas may reflect their anatomical location: a slow-growing tumor might be expected to produce symptoms earlier in the closed intracranial space compared with a tumor developing from the sacrum. Alternatively, there may be an intrinsic biological basis for the earlier initiation of chordomas in the skull base versus the sacrum/coccyx, and it seems plausible that those patients with a genetic predisposition to chordoma would have the earliest onset.

Early age at diagnosis is a hallmark of cancers associated with rare deleterious germline mutations in cancer-predisposition genes, e.g., BRCA1/2, TP53, and CDKN2A. ${ }^{19-22}$ Another feature of such cancers is the presence of disease in both of the paired susceptible organs or at multiple sites. Among the patients reported here, 2 unrelated $T$ - $d u p+$ individuals had at least 1 neuraxial lesion in addition to their presenting chordoma that was considered a second primary chordoma based on anatomical location and imaging characteristics. Because these lesions were asymptomatic, they were not biopsied. A third T-dup+ patient with multicentric chordomas has been reported. ${ }^{3}$ In family 3 , the young girl who was diagnosed and treated for skull base chordoma at the age of 3 years developed a sacral chordoma at age 6 years that was incompletely resected and treated with radio- and chemotherapy. The tumor recurred when she was 19 and again at 25 years; both times she underwent palliative debulking and RT. She died at the age of 28 years, but her cause of death is unknown. ${ }^{3}$

BNCTs are believed to be chordoma precursors. Like chordoma they are thought to derive from notochord remnants and have the same immunohistopathological and molecular profiles. In a few instances, chordomas have been reported to develop from a BNCT. ${ }^{23}$ With a reported incidence of $20 \%$, BNCTs rarely develop into chordomas. However, in the presence of soft-tissue extension, care must be taken to distinguish benign from malignant lesions. The presence of at least 1 BNCT in 5 (38.5\%) $T$ $d u p+$ patients should be considered another manifestation associated with $T$-dup+.

Presenting signs, symptoms, and size of the familial skull base chordomas reported here differed by $T$-dup status. Based on very small numbers, the $T$-dup+ skull base chordomas diagnosed because of symptoms were smaller (median $4.0 \mathrm{~cm}$ vs $5.0 \mathrm{~cm}$ in maximum diameter) and diagnosed later after symptom onset (median 24 months vs 7 months) than symptomatic $T$-dup- tumors. These differences may reflect the direction of tumor growth from the clivus; most $T$-dup+ chordomas grew toward the nasopharynx, which resulted in more nonspecific symptoms than those from $T$ - $d u p$ - chordomas that grew in other directions and impinged cranial nerves. The differences in size and growth patterns between the 2 groups of familial chordomas might explain treatment differences. Surgery alone (either radical resection or GTR) was the main treatment for most $(10 / 13,76.9 \%) T-d u p+$ skull base chordomas, whereas all $T$-dup - skull base chordomas underwent adjuvant fractionated RT after surgery. Current treatment standards for patients with sporadic chordoma recommend postsurgical adjuvant RT if en bloc resection or negative tumor margins have not been achieved. ${ }^{24,25}$ Because optimal surgical outcomes depend in part on tumor location and extent of local invasion, complete pathological resection is often difficult to achieve for skull base (location) and large sacral (local invasion) chordomas.

This study was limited by the small number of chordoma families available for evaluation and the relatively small numbers of nonwhite chordoma patients reported to SEER. In addition, clinical and follow-up details were not available for all family members. Furthermore, given the scope of data collection in SEER, it was not possible to conduct comparisons for treatment, multiple primary lesions, or BNCTs. However, although limited numbers of families have been reported to date, many more families probably exist, and patients would therefore benefit from collection of family history information at chordoma diagnosis to allow for screening and surveillance recommendations. In addition, given the differences observed between white and nonwhite SEER chordoma patients, future studies should explore racial differences in age at diagnosis and presenting site in chordoma.

\section{Conclusions}

The findings from the current study indicate that young age at diagnosis, skull base presentation, or the presence of multiple primary chordomas should encourage careful review of family history for patients diagnosed with chordoma. Identification of a positive family history presents the opportunity for long-term screening of at-risk family members by MRI for early detection of chordoma. In addition, given the relevance of genetic predisposition in some familial chordoma patients, ${ }^{6,8}$ identification of a specific mutation in a family will permit surveillance to be limited to mutation carriers. Finally, consideration should be given for regularly imaging the entire neuraxis in any chordoma patient who presents at an early age or who has a blood relative with chordoma.

\section{Acknowledgments}

We are indebted to the participating families, whose generosity and cooperation have made this study possible. We acknowledge the clinical contributions to this work that were made by Dr. Gladys Glenn. This work was supported by the Intramural Research Program of the Division of Cancer Epidemiology and Genetics, National Cancer Institute, National Institutes of Health.

\section{References}

1. McMaster ML, Goldstein AM, Bromley CM, et al. Chordoma: incidence and survival patterns in the United States, 1973-1995. Cancer Causes Control. 2001;12(1):1-11.

2. Smoll NR, Gautschi OP, Radovanovic I, et al. Incidence and relative survival of chordomas: the standardized mortality ratio and the impact of chordomas on a population. Cancer. 2013;119(11):2029-2037.

3. Bhadra AK, Casey AT. Familial chordoma. A report of two cases. J Bone Joint Surg Br. 2006;88(5):634-636.

4. Enin IP. Chordoma of the nasopharynx in 2 members of a family. Article in Russian. Vestn Otorinolaringol. 1964;26:88-90.

5. Foote RF, Ablin G, Hall WW. Chordoma in siblings. Calif Med. 1958;88(5):383-386.

6. Kelley MJ, Shi J, Ballew B, et al. Characterization of T gene sequence variants and germline duplications in familial and sporadic chordoma. Hum Genet. 2014;133(10):1289-1297. 
7. Wang KE, Wu Z, Tian K, et al. Familial chordoma: a case report and review of the literature. Oncol Lett. 2015;10(5):2937-2940.

8. Yang XR, Ng D, Alcorta DA, et al. T (brachyury) gene duplication confers major susceptibility to familial chordoma. Nat Genet. 2009;41(11):1176-1178.

9. SEER*Stat Database. Incidence - SEER 18 regs research data + Hurricane Katrina impacted Louisiana cases, Nov 2017 sub (1973-2015 varying) - linked to county attributes - total U.S. 1969-2016 counties. National Cancer Institute. Accessed April 22, 2020. https://seer.cancer.gov/data-software/ documentation/seerstat/nov2017/

10. SEER*Stat Software. National Cancer Institute. Accessed April 22, 2020. https://seer.cancer.gov/seerstat

11. Chetty R, Levin CV, Kalan MR. Chordoma: a 20-year clinicopathologic review of the experience at Groote Schuur Hospital, Cape Town. J Surg Oncol. 1991;46(4):261-264.

12. Coppens JR, Ric Harnsberger H, Finn MA, et al. Oronasopharyngeal chordomas. Acta Neurochir (Wien). 2009;151(8):901-907.

13. Dalprà L, Malgara R, Miozzo M, et al. First cytogenetic study of a recurrent familial chordoma of the clivus. Int $J$ Cancer. 1999;81(1):24-30.

14. Kelley MJ, Korczak JF, Sheridan E, et al. Familial chordoma, a tumor of notochordal remnants, is linked to chromosome 7q33. Am J Hum Genet. 2001;69(2):454-460.

15. Kerr WA, Allen KL, Haynes DR, Sellars SL. Familial nasopharyngeal chordoma. Letter. S Afr Med J. 1975;49(39):1584.

16. Miozzo M, Dalprà L, Riva $\mathrm{P}$, et al. A tumor suppressor locus in familial and sporadic chordoma maps to $1 \mathrm{p} 36$. Int J Cancer. 2000;87(1):68-72.

17. Stepanek J, Cataldo SA, Ebersold MJ, et al. Familial chordoma with probable autosomal dominant inheritance. Am J Med Genet. 1998;75(3):335-336.

18. Yang XR, Beerman M, Bergen AW, et al. Corroboration of a familial chordoma locus on chromosome $7 \mathrm{q}$ and evidence of genetic heterogeneity using single nucleotide polymorphisms (SNPs). Int J Cancer. 2005;116(3):487-491.

19. Aoude LG, Wadt KA, Pritchard AL, Hayward NK. Genetics of familial melanoma: 20 years after CDKN2A. Pigment Cell Melanoma Res. 2015;28(2):148-160.

20. Mai PL, Best AF, Peters JA, et al. Risks of first and subsequent cancers among TP53 mutation carriers in the National Cancer Institute Li-Fraumeni syndrome cohort. Cancer. 2016;122(23):3673-3681.

21. Read J, Wadt KA, Hayward NK. Melanoma genetics. J Med Genet. 2016;53(1):1-14.
22. Turnbull C, Hodgson S. Genetic predisposition to cancer. Clin Med (Lond). 2005;5(5):491-498.

23. Yamaguchi T, Yamato M, Saotome K. First histologically confirmed case of a classic chordoma arising in a precursor benign notochordal lesion: differential diagnosis of benign and malignant notochordal lesions. Skeletal Radiol. 2002;31(7):413-418.

24. Walcott BP, Nahed BV, Mohyeldin A, et al. Chordoma: current concepts, management, and future directions. Lancet Oncol. 2012;13(2):e69-e76.

25. Yolcu Y, Wahood W, Alvi MA, et al. Evaluating the role of adjuvant radiotherapy in the management of sacral and vertebral chordoma: results from a national database. World Neurosurg. 2019;127:e1137-e1144.

\section{Disclosures}

The authors report no conflict of interest concerning the materials or methods used in this study or the findings specified in this paper.

\section{Author Contributions}

Conception and design: Goldstein, Parry. Acquisition of data: Goldstein, Parry, Liebsch, Yang. Analysis and interpretation of data: Goldstein, McMaster, Patronas, Quezado. Drafting the article: Parry. Critically revising the article: all authors. Reviewed submitted version of manuscript: all authors. Approved the final version of the manuscript on behalf of all authors: Goldstein. Statistical analysis: McMaster. Administrative/technical/material support: Parry, Zametkin. Study supervision: Goldstein, Parry, Yang.

\section{Supplemental Information}

Online-Only Content

Supplemental material is available with the online version of the article.

Supplemental Tables and Clinical Note. https://thejns.org/doi/ suppl/10.3171/2020.4.JNS193505.

\section{Correspondence}

Alisa M. Goldstein: NCI/NIH, Bethesda, MD. goldstea@mail.nih. gov. 